\title{
Molecular Gas at High Redshift
}

\author{
Fabian Walter $^{1}$, Chris Carilli ${ }^{2}$ \& Emanuele Daddi ${ }^{3}$ \\ 1 Max Planck Institut für Astronomie, Auf dem Hügel 71, 69117 Heidelberg, Germany \\ 2 National Radio Astronomy Observartory, Socorro, NM 87801, USA \\ ${ }^{3}$ Laboratoire AIM, CEA Saclay, 91191 Gif-sur-Yvette Cedex, France
}

Received 30 May 2005, accepted 11 Nov 2005

Published online later

Key words early universe, galaxies: ISM, galaxies: high redshift, radio lines: ISM

\begin{abstract}
In order to understand galaxy evolution through cosmic times it is critical to derive the properties of the molecular gas content of galaxies, i.e. the material out of which stars ultimately form. The last decade has seen rapid progress in this area, with the detection of massive molecular gas reservoirs at high redshifts in submillimeter-selected galaxies and quasars. In the latter case, molecular gas reservoirs have been quantified out to redshifts $z>6$, i.e. towards the end of cosmic reionization when the universe was less than one Gyr old. The recent discovery of molecular gas in more normal galaxies have extended these studies from the most extreme objects in the unviverse (SFR $\sim 1000 \mathrm{M}_{\odot} \mathrm{yr}^{-1}$; quasars and submillimeter galaxies) to more 'normal' starforming systems at redshifts $1.5-2.5$ (with $\mathrm{SFR} \sim 100 \mathrm{M}_{\odot} \mathrm{yr}^{-1}$ ). However, detecting the molecular gas reservoirs of high-redshift galaxies that only have moderate star formation rates $\left(\sim 10 \mathrm{M}_{\odot} \mathrm{yr}^{-1}, \operatorname{similar}\right.$ to the faint galaxies seen in the Hubble Ultra Deep Field) will likely have to await the completion of ALMA.
\end{abstract}

(C) 2006 WILEY-VCH Verlag GmbH \& Co. KGaA, Weinheim

\section{Recent Progress in understanding Galaxy Formation}

The last decade has seen dramatic advances in our understanding of galaxy formation. Cosmic geometry, the massenergy content of the Universe, and the initial density fluctuation spectrum, have been constrained to high accuracy (e.g., Spergel et al. 2007). Given the underlying distribution of dark matter, the main challenge of galaxy formation studies is to understand how the baryons assemble into the observed Universe. In this context, structure formation through gravitational instabilities has been calculated in detail through numerical studies, and observationally verified through studies of galaxy distributions (e.g., Springel et al. 2006). The history of star formation, and the build up of stellar mass, as a function of galaxy type and mass, are now constrained out to the tail-end of cosmic reionization, within $1 \mathrm{Gyr}$ of the Big Bang, and pushing toward first light in the Universe (e.g., Bouwens et al. 2010).

The principle results of these studies can be summarized as follows. The comoving cosmic star formation rate density increases by more than an order of magnitude from $z \sim 0$ to 1 , peaks around $z \sim 2$ to 3 , and likely drops gradually out to $z \sim 8$ (e.g., Hopkins \& Beacom 2006, Bouwens et al. 2010). The build-up of stellar mass follows this evolution, as does the temporal integral (for $\mathrm{z}<2$, e.g. Ilbert et al. 2010). The redshift range $z \sim 1.5$ to 3 has been called the 'epoch of galaxy assembly', when about half the stars in the Universe form in spheroidal galaxies. It has been shown that star formation shifts systematically from lower luminosity galaxies $\left(\mathrm{L}_{F I R} \sim 10^{10} \mathrm{~L}_{\odot}\right)$ at low redshift, to high star formation rate galaxies $\left(\mathrm{L}_{F I R} \geq 10^{11} \mathrm{~L}_{\odot}\right)$ at $z \sim 2$ ('downsizing', e.g., Le Floc'h et al. 2005, Smolčić et al. 2009). Another key finding is the tight relation between the star formation rate and the stellar mass for star forming galaxies up to redshifts z 3 (e.g. Noeske et al. 2007; Elbaz et al. 2007; Daddi et al. 2007; Magdis et al. 2010). High-redshift starforming galaxies are typically selected through optical/IR studies (e.g., Steidel et al. 1999, Daddi et al. 2004, van Dokkum et al. 2006)

Besides the star forming galaxies, a population of passively evolving, relatively massive (stellar masses $\sim 10^{10}$ to $10^{11} \mathrm{M}_{\odot}$ ) galaxies ('red and dead'), have been detected at $z>1$, comprising roughly $50 \%$ of the galaxies selected in near-IR surveys (see, e.g., review by Renzini 2006). These galaxies must form the majority of their stars quickly at even earlier epochs (e.g., Wiklind et al. 2008).

Lastly, substantial populations of supermassive black holes and galaxies are now being detected routinely back to first light and cosmic reionization $(z \sim 6.5)$. Populations include normal star forming galaxies, such as the Ly- $\alpha$ selected galaxies, with star formation rates of order $10 \mathrm{M}_{\odot}$ year $^{-1}$ (Taniguchi et al. 2005, Ouchi et al. 2009) as well as quasar host galaxies, often with star formation rates that are 100 times higher (e.g., Wang et al 2008). The latter likely represent a major star formation epoch for very massive galaxies within $1 \mathrm{Gyr}$ of the Big Bang. Most recently, GRBs are showing great potential to probe galaxy formation out to $z>8$ (Tanvir et al. 2009, Salvaterra et al. 2009).

These observations have led to a new model for galaxy formation. As opposed to either cooling of virialized, hot halo gas, or major, gas-rich mergers, the dominant mode of 
star formation during the epoch of galaxy assembly may be driven by cold mode accretion, or stream-fed galaxy formation (e.g., Kereš et al. 2005, Dekel et al. 2009). Simulations suggest that gas accretion in early massive galaxies occurs along cold streams from the filamentary intergalactic medium (IGM) that never shock-heat, but streams onto the galaxy at close to the free-fall time. This cool gas forms a thick, turbulent rotating disk which very efficiently forms stars in a few giant regions in the disk (e.g., Bournaud et al. 2007). The star forming regions then migrate to the galaxy center via dynamical friction and viscosity, forming compact stellar bulges. The process leads to relatively steady, active $\left(\sim 100 \mathrm{M}_{\odot}\right.$ year $\left.^{-1}\right)$ star formation in galaxies over timescales of order 1 Gyr. Subsequent dry mergers at $z<2$ lead to continued total mass build up, and morphological evolution, but little subsequent star formation (e.g., Skelton et al. 2009, Van der Wel et al. 2009, Robaina et al. 2010).

\section{Molecular gas: the key to testing galaxy formation models}

While progress has been impressive, the model above is based almost exclusively on optical and near-IR studies of the stars, star formation, and ionized gas. There remains a major gap in our understanding of galaxy formation, namely, observations of the cool gas, the fuel for star formation in galaxies. In essence, current studies probe the products of the process of galaxy formation, but miss the source. $\mathrm{Nu}$ merous observational and theoretical papers have pointed out the crucial need for observations of the cool molecular gas feeding star formation in galaxies (e.g., Dressler et al. 2009; Obreschkow \& Rawlings 2009).

The molecular gas density history of the Universe: The key to future studies of galaxy formation is to determine the evolution of the molecular gas density of the Universe. Over the last two decades, the star formation history of the Universe (SFHU) plot has been perhaps the most fundamental tool for studying galaxy formation. At the same time, very detailed studies of star formation in nearby galaxies have reached two critical conclusions. First, star formation relates closely with the molecular gas content, but has little relation to the atomic neutral gas content (e.g., Bigiel et al. 2009). This fact is verified at high $z$ by the lack of evolution of the cosmic HI mass density, as determined from damped Ly $\alpha$ systems, over the same redshift range where the star formation rate density is increasing by more than an order of magnitude (Prochaska \& Wolfe 2009). Second, once molecular gas forms, it forms stars, to first order, according to a star formation law (e.g., Kennicutt 1998, Bigiel et al. 2009, Leroy et al. 2009, Krumholz et al. 2009, Daddi et al. 2010b, Genzel et al. 2010). In low-density environemnts, the general idea is that, once a giant molecular cloud becomes self-gravitating, star formation proceeds via local processes inherent to the GMC. If the universal star formation law were to hold to high redshift, then the classic SFHU plot would just be a reflection of the molecular gas density history of the Universe.

The stellar to gas mass density ratio: A related, and similarly fundamental issue is to compare the stellar and gas mass of galaxies versus redshift. Two extremes scenarios can be envisioned. At one extreme, the gas builds up to large values (approaching $10^{11} \mathrm{M}_{\odot}$ ), and then a dramatic starburst is triggered via, e.g., a major gas rich merger, when the gas is rapidly converted into stars on dynamical timescales $\sim 10^{8}$ years. At the other extreme, a continuous supply of gas comes from the IGM, cooling into molecular clouds and forming stars at a rate comparable to the free-fall rate of gas onto the galaxy over $\sim$ Gyr timescales. This latter case corresponds to the cold mode accretion model. This point is currently addressed by a number of observational programs that aim at studying star forming galaxies at $\mathrm{z} \sim 2$ (Daddi et al. 2010a, 2010b, Tacconi et al. 2010, Genzel et al. 2010, as discussed in Sec. 4.3 and 4.4 below).

More generally, the study of the cool molecular gas phase in 'typical' high high-redshift galaxies (with SFR $100 \mathrm{M}_{\odot} \mathrm{yr}^{-1}$ ) provide key insight into the process of galaxy formation in a number of ways:

- A universal star formation law: The low order transitions of $\mathrm{CO}$ provide the most direct means of measuring the total molecular gas mass. These measurements can then be compared to star formation rates to determine the evolution of the fundamental star formation laws relating cool gas and star formation. Is there a universal relationship that governs the efficiency of conversion of molecular gas to stars in galaxies of various types through cosmic time? What are the fundamental physical parameters behind the relationship? For a first attempt to address this topic see Sections 4.3 and 4.4 below.

- How does the ratio of molecular gas to stellar mass evolve? This ratio is typically $<0.1$ in the nearby Universe, even for gas rich galaxies such as the Milky Way. Observations of a few the $\mathrm{sBzK}$, and $\mathrm{BX} / \mathrm{BM}$ galaxies provide evidence that this ratio may increase to $>1$ at $z \sim 2$ (Daddi et al. 2010a, Tacconi et al. 2010).

- Galaxy dynamics and star formation: Molecular line observations are the most direct means of studying galaxy dynamics. Are these systems dominated by rotating disks, as predicted by cold mode accretion, or major mergers? What is the relative magnitude of ordered motion vs. turbulence? What are the stability criteria, and how do they compare to the distribution of star formation?

- Black hole - bulge mass relation: Establishing a galaxy's mass through molecular gas dynamics can be used to test the evolution of the black hole - bulge mass relation back to the first galaxies. Indeed, in many cases, the molecular line observations are the only means to get galaxy dynamics for galaxies with optically bright AGN (e.g., Walter et al. 2003, Shields et al. 2006, Riechers et al. 2008, 2009). The local relation suggests that the formation of black holes and galaxies are closely tied, 
but the origin of this relationship remains a mystery (see Sec. 4.2).

- ISM physics and chemistry: Detailed studies of the physical conditions in the ISM of primeval galaxies can be performed using numerous molecular line transitions, including excitation studies of $\mathrm{CO}$, observations of dense gas tracers like $\mathrm{CN}, \mathrm{HCN}$ and $\mathrm{HCO}^{+}$, and other key astrochemical tracers. Such observations determine the temperature and density of the ISM, molecular abundances and the fraction of dense gas. To date, only a few extreme high-redshift systems have been detected in these dense gas tracers (e.g. Vanden Bout et al. 2003, Riechers et al. 2006, 2007a, 2007b, Garcia-Burillo et al. 2006)

\section{Recent progress on molecular lines observations in early galaxies}

\subsection{Submillimeter Galaxies (SMGs)}

Surveys at submm wavelengths have revealed highly obscured, extreme starburst galaxies $\left(\mathrm{SFR}>1000 \mathrm{M}_{\odot}\right.$ year $\left.^{-1}\right)$ at high redshift, the so-called submillimeter galaxies (SMGs, e.g., Smail et al. 2007, Blain et al. 2002). Subsequent studies have detected large reservoirs of molecular gas in these systems $\left(>10^{10} \mathrm{M}_{\odot}\right.$; e.g., Frayer et al. 1998, 1999, Genzel et al. 2003, Greve et al. 2005, Tacconi et al. 2006, 2008, Knudsen et al. 2009, Ivison et al. 2010). General properties of the SMGs are summarized in the review by Solomon \& Vanden Bout (2005) and the detections to date are summarized in Figure 1 (as discussed in Sec. 4.3).

High-resolution imaging of SMGs by Tacconi et al. (2006, 2008) have shown that their molecular gas reservoirs are compact, with a median diameter of $\sim 4 \mathrm{kpc}$. This morphology can be explained if these galaxies are the results of mergers, in which the molecular gas settles in the centre of two interacting galaxies (leading to a starburst). Recent $\mathrm{CO}$ imaging of one of the most distant SMGs suggests that cold mode accretion may also play a role in powering the ongoing star formation (Carilli et al. 2010).

Chapman et al. (2005) have shown that the median redshift of SMGs is $\mathrm{z} \sim 2.3$ - the existence of a substantial high-redshift $(z>4)$ tail of the submillimeter galaxy population that host molecular gas has recently been established by a number of studies (Schinnerer et al. 2009, Daddi et al. 2009a, 2009b, Carilli et al. 2010, Coppin et al. 2010).

While the study of these SMGs has opened a new window on the optically obscured Universe at high redshift, the fact remains that these sources are rare, likely representing the formation of the most massive galaxies in mergerdriven hyper-starbursts at high redshift, with gas depletion timescales $<0.1 \mathrm{Gyr}$,

\subsection{Quasars}

Likewise, surveys of molecular gas in quasar host galaxies have revealed the presence of massive reservoirs of molec- ular gas in these objects $\left(>10^{10} \mathrm{M}_{\odot}\right.$, i.e. comparable to the submillimeter galaxies, e.g., Solomon \& Vanden Bout 2005, Coppin et al. 2008). Quite remarkably, such reservoirs have now been detected in a sizable sample of quasars at redshift 6 and greater, i.e. at the end of cosmic reionization when the Universe was less than one Gyr old (Walter et al. 2003, 2004, Bertoldi et al. 2003, Maiolino et al. 2007, Carilli et al. 2007, Wang et al. 2010). Resolved imaging of a few quasar hosts (Walter et al. 2004, Riechers et al. 2008a, b, Riechers et al. 2009) imply that the molecular gas is extended on kpc scales around the central black hole. It remains puzzling how such amounts of cold molecular gas, rich in $\mathrm{C}$ and $\mathrm{O}$, can be distributed over many kpc scales on short (few 100 Myr) timescales.

The high FIR luminosity of the quasars, in conjunction with their massive molecular gas reservoirs imply high star formation rates in the host galaxies of SFR $>1000$ $\mathrm{M}_{\odot}$ year $^{-1}$. This finding is corroborated by the detection of [CII] emission in a quasar host at $\mathrm{z}=6.42$ (Maiolino et al. 2005, Walter et al. 2009). In fact, spatially resolved measurements of the [CII] emission were able to constrain the size of the starburst to be emerging from the central $\sim 1.5 \mathrm{kpc}$ of the quasar host. This implies extremely high star formation rate surface densities $\left(\sim 1000 \mathrm{M}_{\odot} \mathrm{yr}^{-1} \mathrm{kpc}-2\right)$, at the maximum what is allowed theoretically based on Edingtonlimited star formation of a radiation-pressure-supported starburst (Walter et al. 2009a).

The central black hole masses of the quasars that are currently detectable reach masses of many $10^{9} \mathrm{M}_{\odot}$. If the local relation seen between the central black hole and surrounding bulge mass (e.g., Magorrian et al. 1998, Ferrarese et al. 2000, Gebhardt et al. 2000) were to hold at these high redshifts, one would expect bulge masses of order $10^{12} \mathrm{M}_{\odot}$. Such high masses exceed dynamical mass estimates of a few sources by more than an order of magnitude (Walter et al. 2004, Riechers et al. 2008a,b, 2009), which in turn indicates that black holes built up their masses more quickly than the stellar bulges (at least in some quasars).

\section{3 'Normal' starforming galaxies}

More recently, major progress has been made on detecting $\mathrm{CO}$ emission from more normal star forming galaxies at high $z$. These come in the form of near-IR and color selected galaxy samples at $z \sim 1.5$ to 3 . The $\mathrm{BzK}$ color selection technique in particular has revealed a sample of star forming galaxies at $z \sim 1.5$ to 2.5 , selected at near-IR wavelengths, with stellar masses in the range $10^{10}$ to $10^{11} \mathrm{M}_{\odot}$, and star formation rates $\sim 100 \mathrm{M}_{\odot} \mathrm{yr}^{-1}$ (Daddi et al. 2004). These galaxies have a space density $>10$ times those the submm galaxies.

Daddi et al. (2008) showed that star forming BzK galaxies (sBzK) uniformly contain molecular gas reservoirs of comparable mass to the submm galaxies, and yet they are forming stars at $\sim 10$ times lower rates. This leads to high gas depletion times of up to $\sim 1$ Gyr (see Fig. 1). Followup observations showed that massive gas reservoirs have 

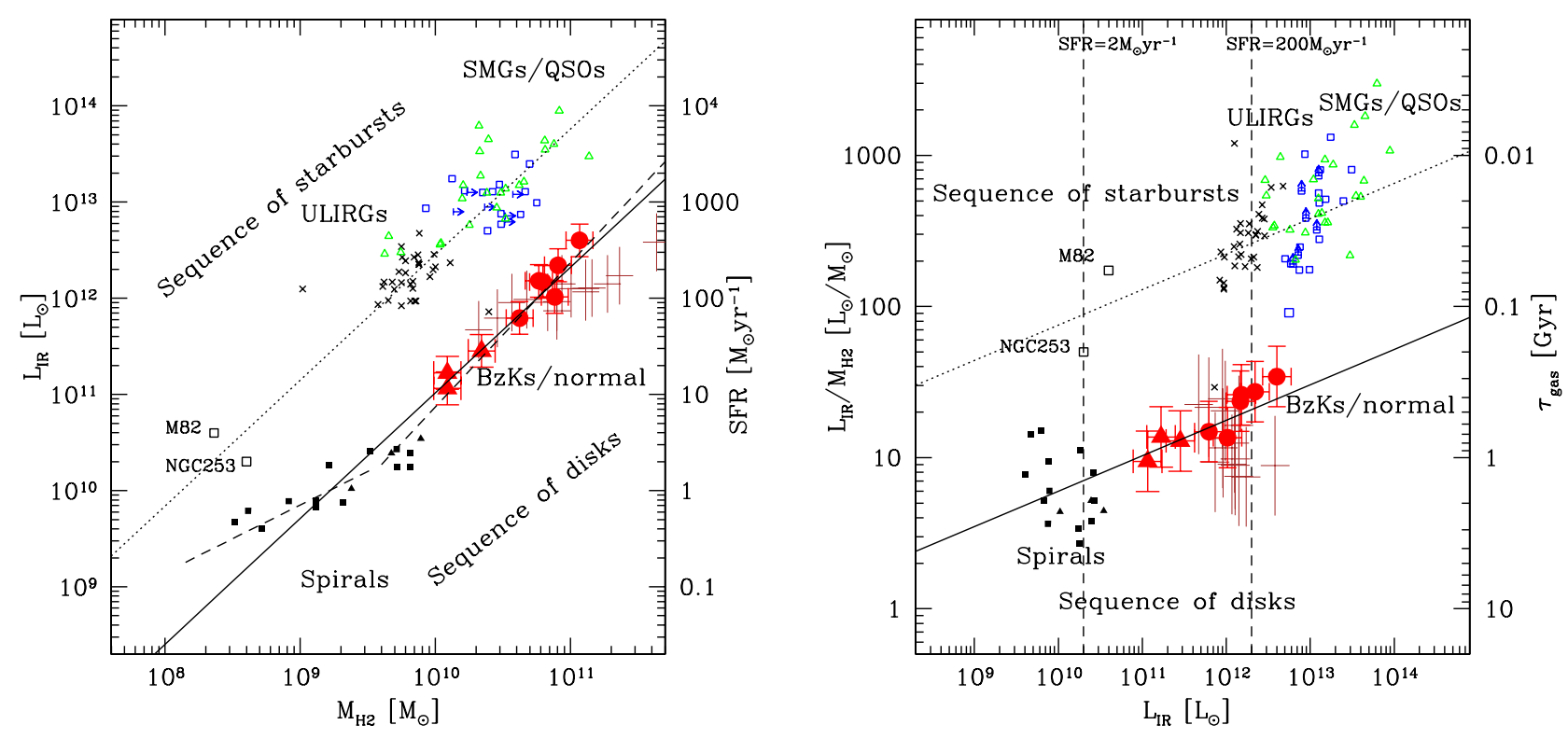

Fig. 1 Comparison of molecular gas masses and total IR bolometric luminosities (taken from Daddi et al. 2010b) for all high-redshifted systems detected in CO to date: BzK galaxies (red filled circles; Daddi et al. $2010 \mathrm{a}$ ), $z \sim 0.5$ disk galaxies (red filled triangles; Salmi et al. in prep.), $z=1-2.3$ normal galaxies (Tacconi et al. 2010; brown crosses), SMGs (blue empty squares; Greve et al. 2005, Frayer et al. 2005, Daddi et al. 2009a, 2009b), QSOs (green triangles; Riechers et al. 2006, Solomon \& Vanden Bout 2005), local ULIRGs (black crosses; Solomon et al. 1997), local spirals (black filled square: Leroy et al. 2009; black filled triangles: Wilson et al. 2009). The two nearby starbursts M82 and the nucleus of NGC 253 are also shown (see Daddi et al. 2010b for references). The solid line (slope of 1.31 in the left panel) is a fit to local spirals and BzK galaxies and the dotted line is the same relation shifted in normalization by 1.1 dex. For guidance, two vertical lines indicate $S F R=2$ and $200 \mathrm{M}_{\odot} \mathrm{yr}^{-1}$ in the right panel. For more details see Daddi et al. (2010b).

been detected in each BzK galaxy that was targeted (Daddi et al. 2010a). The implied gas fractions are very high and the gas mass in these galaxies is comparable to or larger than the stellar mass, and the gas accounts for 50-65\% of the baryons with the galaxies' half-light radius. New observations of an even larger sample of 'normal' starforming galaxies have confirmed and extended these findings (Tacconi et al. 2010).

In a few cases, the molecular gas emission in these objects could even be spatially resolved (see Tacconi et al. 2010, Fig. 2, for a particularly striking example) allowing for a determination of the dynamical mass. A good constraint on the dynamical mass also allows one to derive an independent measurement of the $\mathrm{CO}-$ to $-\mathrm{H}_{2}$ conversion factor (as done in the BzK sample by Daddi et al. 2010a). The interesting result of such an analysis is that the BzK's have a conversion factor similar to the Galaxy, i.e. a factor of $\sim 5$ larger than what is found in local ULIRGs.

In conclusion these systems likely represent the major star formation epoch in early massive galaxies (presumably driven by cold mode accretion). In the current picture these galaxies may turn into passively evolving galaxies at $z \sim 1$, which may eventually turn into 'red and dead' cluster elliptical galaxies seen today. They are substantially different from the quasar and submillimeter population discussed above in terms of their larger spatial sizes, lower star for- mation efficiencies, and lower molecular gas excitation (e.g. Dannerbauer et al. 2009, Aravena et al. 2010).

\subsection{Different Star Formation Laws at High Redshift?}

The detection of molecular gas in systems spanning a wide range of parameters now allows one to investigate the star formation law (i.e. the dependence of the star formation rate on the available gas) at high redshift. In Figure 1 (left), we show the summary plot of Daddi et al. 2010b. Here the infrared luminosities (a proxy for the SFR) is plotted as function of molecular gas mass $\left(\mathrm{M}_{\mathrm{H} 2}\right)$ of all high-redshift objects detected in $\mathrm{CO}$ to date, including submillimeter galaxies, quasars and BzK,BM/BX galaxies (see figure caption for references). As discussed in Daddi et al. (2010b), two different conversion factors (to derive $\mathrm{M}_{\mathrm{H} 2}$ ) have been used for the high redhift galaxy populations in this plot: the Galactic one for the local spiral galaxies and the BzK/normal galaxies at $\mathrm{z} \sim 1.5-2.5$ and a lower 'ULIRG' conversion factor for the ULIRS, submillimeter galaxies and quasars. Plotted this way, there are two sequences emerging (each of which can be fitted with a power law of slope $\sim 1.3$ ), one for the 'starbursts' (ULRIGS, SMGs and QSOs) and one for the 'disk' galaxies. It should be stressed that the offset in sequences seen here is not only due to the choice of conversion factors (e.g., Fig. 13 in Daddi et al. 2010a). The 
right hand panel shows the same data, but the y-axis shows the ratio of infrared luminosity to molecular gas mass (i.e. the the gas depletion time) plotted as a function of total infrared luminosity. It is obvious from this figure that the gas depletion times in the BzKs/normal galaxies are about an order of magnitude longer than in the systems that are presumably undergoing mergers (such as the ULIRGs, SMGs and QSOs; see also discussion in Genzel et al. 2010). These different star formation efficiencies appear to be regulated by the dynamical timescales of the systems (Daddi et al. 2010b, Genzel et al. 2010).

These initial molecular gas observations of the different population of star forming galaxies during the epoch of galaxy assembly present a remarkable opportunity to study the formation of normal galaxies at critical early epochs, when most of their stars form, and when the dominant baryon mass constituent was gas, rather than stars. The galaxies are typically extended on scales $\sim 1$ " in the optical, providing ideal targets for follow-up high resolution imaging of the gas using the EVLA and ALMA.

\section{Outlook: EVLA and ALMA}

Major progress in the studies of the molecular gas content of galaxies throughout cosmic times is currently limited by the following technological restrictions:

- Sensitivity: Although remarkable progress has been made in recent years, the sensitivities of current interferometers do only allow to study the molecular gas content in objects that have high star formation rates and/or high molecular gas masses (of order $10^{10} \mathrm{M}_{\odot}$, SFR $\sim 100 \mathrm{M}_{\odot}$ y To push this to more typical object at high redshift (with SFR that are an order of magnitude lower) much higher sensitivites are required. ALMA, with its unprecedented collecting area, will without doubt revolutionize the field of quantifying the molecular gas content in high-z systems, e.g. through dedicated studies of molecular line deep fields.

- Resolution: Spatially resolved imaging of galaxies remains an important task to derive the extent of the molecular gas emission and to constrain the dynamical masses of the systems (which in turn give independent estimates of the conversion factor to derive molecular gas masses from CO observations), and to search for observational evidence for interactions and/or cold mode accretion. Both EVLA and ALMA will enable such studies at high spatial resolution - but even with these new facilities such studies will be restricted to small samples due to the intrinsic faintness of the sources.

- Spectral Coverage: As galaxies are observed at higher and higher redshifts, their molecular lines are redshifted. Since the molecular gas excitation has proven to be different in quasars, submillimeter galaxies and more normal galaxies (e.g., Weiss et al. 2005, 2007a, 2007b, Dannerbauer et al. 2009), it is important that the same tranisions (e.g. rotational J transitions of carbon monoxide) are observed to compare the various galaxy populations. The EVLA will enable detailed studies of the ground transitions of $\mathrm{CO}$ up to high redshift. On the other hand ALMA will enable studies of molecular gas tracers that are currently very difficult to observe (e.g. [CII], [NII], [OIII] and other fine structure lines, e.g., Walter et al. 2009b).

Acknowledgements. It is our pleasure to acknowledge our collaborators, in particular Frank Bertoldi, Pierre Cox, Helmut Dannerbauer, Roberto Maiolino, Roberto Neri, Dominik Riechers, Ran Wang, and Axel Weiss.

\section{References}

Aravena, M., et al. 2010, ApJ, in press

Bigiel, F., Leroy, A., Walter, F., Brinks, E., de Blok, W. J. G., Madore, B., \& Thornley, M. D. 2008, AJ, 136, 2846

Blain, A. W., Smail, I., Ivison, R. J., Kneib, J.-P., \& Frayer, D. T. 2002, PhysRep, 369, 111

Bournaud, F., Elmegreen, B. G., \& Elmegreen, D. M. 2007, ApJ, 670,237

Bouwens, R. J., et al. 2010, ApJL, 709, L133

Carilli, C. L., et al. 2010, arXiv:1002.3838

Carilli, C. L., et al. 2007, ApJL, 666, L9

Chapman, S. C., Blain, A. W., Smail, I., \& Ivison, R. J. 2005, ApJ , 622,772

Coppin, K. E. K., et al. 2008, MNRAS, 389, 45 Coppin et al. 2010, MNRAS, to be submitted.

Daddi, E., et al. 2010a, ApJ, 713, 686

Daddi, E., et al. 2010b, arXiv:1003.3889

Daddi, E., Dannerbauer, H., Krips, M., Walter, F., Dickinson, M., Elbaz, D., \& Morrison, G. E. 2009b, ApJL, 695, L176

Daddi, E., et al. 2009a, ApJ, 694, 1517

Dde i, E., et al. 2007, ApJ , 670, 156

Daddi, E., Dannerbauer, H., Elbaz, D., Dickinson, M., Morrison, G., Stern, D., \& Ravindranath, S. 2008, ApJL, 673, L21

Daddi, E., Cimatti, A., Renzini, A., Fontana, A., Mignoli, M., Pozzetti, L., Tozzi, P., \& Zamorani, G. 2004, ApJ, 617, 746

Dannerbauer, H., Daddi, E., Riechers, D. A., Walter, F., Carilli, C. L., Dickinson, M., Elbaz, D., \& Morrison, G. E. 2009, ApJL, 698, L178

Dekel, A., et al. 2009, Nature, 457, 451

Dressler, A., Oemler, A., Gladders, M. G., Bai, L., Rigby, J. R., \& Poggianti, B. M. 2009, ApJL, 699, L130

Elbaz, D., et al. 2007, A\&A, 468, 33

Ferrarese, L., \& Merritt, D. 2000, ApJL, 539, L9

Frayer, D. T., et al. 2008, ApJ , 680, L21

Frayer, D. T., et al. 1999, ApJ , 514, L13

Frayer, D. T., Ivison, R. J., Scoville, N. Z., Yun, M., Evans, A. S., Smail, I., Blain, A. W., \& Kneib, J.-P. 1998, ApJ , 506, L7

García-Burillo, S., et al. 2006, ApJ , 645, L17

Gebhardt, K., et al. 2000, ApJL, 539, L13

Genzel, R., Baker, A. J., Tacconi, L. J., Lutz, D., Cox, P., Guilloteau, S., \& Omont, A. 2003, ApJ, 584, 633

Genzel, R., et al. 2010, arXiv:1003.5180

Greve, T. R., et al. 2005, MNRAS, 359, 1165

Hopkins, A. M., \& Beacom, J. F. 2006, ApJ, 651, 142

Ilbert, O., et al. 2010, ApJ , 709, 644

Ivison, R. J., Smail, I., Papadopoulos, P. P., Wold, I., Richard, J., Swinbank, A. M., Kneib, J.-P., \& Owen, F. N. 2010, MNRAS, 261

Kennicutt, R. C., Jr. 1998, ApJ , 498, 541 
Kereš, D., Katz, N., Weinberg, D. H., \& Davé, R. 2005, MNRAS, 363,2

Knudsen, K. K., Neri, R., Kneib, J.-P., \& van der Werf, P. P. 2009 , A\&A, 496, 45

Krumholz, M. R., McKee, C. F., \& Tumlinson, J. 2009, ApJ, 699, 850

Le Floc'h, E., et al. 2005, ApJ, 632, 169

Leroy, A. K., Walter, F., Brinks, E., Bigiel, F., de Blok, W. J. G., Madore, B., \& Thornley, M. D. 2008, AJ, 136, 2782

Magdis, G. E., Rigopoulou, D., Huang, J.-S., \& Fazio, G. G. 2010, MNRAS , 401, 1521

Magorrian, J., et al. 1998, AJ, 115, 2285

Maiolino, R., et al. 2007, A\&A, 472, L33

Maiolino, R., et al. 2005, A\&A, 440, L51

Noeske, K. G., et al. 2007, ApJL, 660, L43

Obreschkow, D., \& Rawlings, S. 2009, ApJL, 696, L129

Ouchi, M., et al. 2009, ApJ, 696, 1164

Prochaska, J. X., \& Wolfe, A. M. 2009, ApJ, 696, 1543

Renzini, A. 2006, ARA\&A, 44, 141

Riechers, D. A., et al. 2006, ApJ, 650, 604

Riechers, D. A., Walter, F., Carilli, C. L., Weiss, A., Bertoldi, F., Menten, K. M., Knudsen, K. K., \& Cox, P. 2006, ApJL, 645, L13

Riechers, D. A., Walter, F., Cox, P., Carilli, C. L., Weiss, A., Bertoldi, F., \& Neri, R. 2007a, ApJ, 666, 778

Riechers, D. A., Walter, F., Carilli, C. L., \& Bertoldi, F. 2007b, ApJL, 671, L13

Riechers, D. A., Walter, F., Carilli, C. L., Bertoldi, F., \& Momjian, E. 2008a, ApJL, 686, L9

Riechers, D. A., Walter, F., Brewer, B. J., Carilli, C. L., Lewis, G. F., Bertoldi, F., \& Cox, P. 2008b, ApJ, 686, 851

Riechers, D. A., et al. 2009, ApJ, 703, 1338

Robaina, A. R., Bell, E. F., van der Wel, A., Somerville, R. S., Skelton, R. E., McIntosh, D. H., Meisenheimer, K., \& Wolf, C. 2010 , arXiv: 1002.4193

Salvaterra, R., et al. 2009, Nature, 461, 1258

Schinnerer, E., et al. 2008, ApJL, 689, L5

Shields, G. A., Menezes, K. L., Massart, C. A., \& Vanden Bout, P. 2006, ApJ , 641, 683

Skelton, R. E., Bell, E. F., \& Somerville, R. S. 2009, ApJ , 699, L9

Solomon, P. M., Downes, D., Radford, S. J. E., \& Barrett, J. W. 1997, ApJ , 478, 144

Solomon, P. M., \& Vanden Bout, P. A. 2005, ARA\&A, 43, 677

Spergel, D. N., et al. 2007, ApJS, 170, 377

Springel, V., Frenk, C. S., \& White, S. D. M. 2006, Nature, 440, 1137

Steidel, C. C., Adelberger, K. L., Giavalisco, M., Dickinson, M., \& Pettini, M. 1999, ApJ , 519, 1

Smail, I., Ivison, R. J., \& Blain, A. W. 1997, ApJL, 490, L5

Smolčić, V., et al. 2009, ApJ, 690, 610

Tacconi, L. J., et al. 2010, Nature, 463, 781

Tacconi, L. J., et al. 2008, ApJ, 680, 246

Tacconi, L. J., et al. 2006, ApJ, 640, 228

Taniguchi, Y., et al. 2005, PASJ, 57, 165

Tanvir, N. R., et al. 2009, Nature, 461, 1254

Vanden Bout, P. A., Solomon, P. M., \& Maddalena, R. J. 2004, ApJ , 614, L97

van der Wel, A., Rix, H.-W., Holden, B. P., Bell, E. F., \& Robaina, A. R. 2009, ApJ , 706, L120

van Dokkum, P. G., et al. 2006, ApJ , 638, L59

Walter, F., Riechers, D., Cox, P., Neri, R., Carilli, C., Bertoldi, F., Weiss, A., \& Maiolino, R. 2009, Nature, 457, 699
Walter, F., Weiß, A., Riechers, D. A., Carilli, C. L., Bertoldi, F., Cox, P., \& Menten, K. M. 2009, ApJL, 691, L1

Walter, F., Carilli, C., Bertoldi, F., Menten, K., Cox, P., Lo, K. Y., Fan, X., \& Strauss, M. A. 2004, ApJL, 615, L17

Walter, F., et al. 2003, Nature, 424, 406

Wang, R., et al. 2008, ApJ, 687, 848

Wang, R., et al. 2010, arXiv:1002.1561

Weiss, A., Downes, D., Walter, F., \& Henkel, C. 2007a, From ZMachines to ALMA: (Sub)Millimeter Spectroscopy of Galaxies, 375, 25

Weiß, A., Downes, D., Neri, R., Walter, F., Henkel, C., Wilner, D. J., Wagg, J., \& Wiklind, T. 2007b, A\&A, 467, 955

Weiß, A., Downes, D., Walter, F., \& Henkel, C. 2005, A\&A, 440, L45

Wilson, C. D., et al. 2009, ApJ , 693, 1736

Wiklind, T., Dickinson, M., Ferguson, H. C., Giavalisco, M., Mobasher, B., Grogin, N. A., \& Panagia, N. 2008, ApJ, 676, 781 